\title{
Gradhiva
}

GRADHIV

Revue d'anthropologie et d'histoire des arts

15 | 2012

Robots étrangement humains

\section{Le Bouddha dans le robot}

Rencontre avec le professeur Masahiro Mori

\section{CpenEdition}

\section{Journals}

Édition électronique

URL : http://journals.openedition.org/gradhiva/2393

DOI : 10.4000/gradhiva.2393

ISSN : 1760-849X

\section{Éditeur}

Musée du quai Branly Jacques Chirac

Édition imprimée

Date de publication : 16 mai 2012

Pagination : 162-181

ISBN : 978-2-35744-047-0

ISSN : 0764-8928

\section{Référence électronique}

«Le Bouddha dans le robot », Gradhiva [En ligne], 15 | 2012, mis en ligne le 16 mai 2015, consulté le 01 mai 2019. URL : http://journals.openedition.org/gradhiva/2393; DOI : 10.4000/gradhiva.2393

(c) musée du quai Branly 



\section{Rencontre avec le professeur Masahiro Mori}

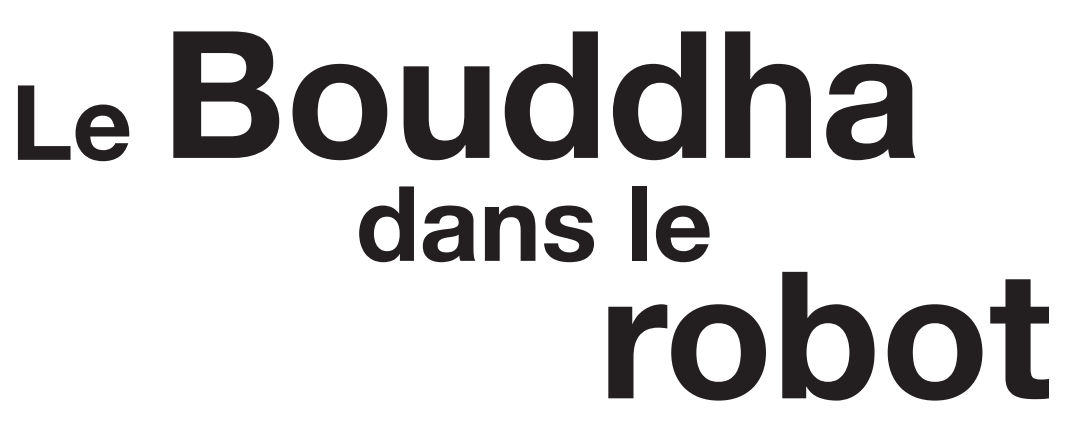

Fig. 1 Main de Miroku Bosatsu au temple de Koryu-ji, Kyoto. DR.

- -

1. Zaven Paré et Emmanuel Grimaud tiennent à remercier pour sa collaboration inestimable Chihiro Minato, anthropologue et photographe (université de Tama, Tokyo) et qui a été ici bien plus qu'un interprète.

2. L'intégralité de la rencontre, filmée par Emmanuel Grimaud, est disponible sur le site de Gradhiva en version originale.
Ce texte est le fruit d'un entretien inédit avec le professeur Masahiro Mori, pionnier de la robotique japonaise. C'est à l'automne 2009 que Zaven Paré, Emmanuel Grimaud et Chihiro Minato ont rencontré le théoricien de l'uncanny valley à Tokyo ${ }^{1}$. Au cours de cette rencontre, dont l'intégralité a été filmée ${ }^{2}$, est évoquée l'histoire de la robotique japonaise au moment crucial de la fusion des biotechnologies avec l'ingénierie mécanique, notamment sous l'impulsion de la création du Mukta Research Institute. En 1974, Mori a aussi publié The Buddha in the robot. A robot engineer's thoughts on science and religion, dans lequel il évoque les implications métaphysiques de la robotique. Il est le fondateur du concours de robotique RoboCon, qui a débuté en 1981. Il est aussi le "grand-père " de la plupart des robots japonais, y compris Asimo, le robot humanoïde de Honda, qui a été développé par Toru Takenaka, l'un de ses étudiants.

Zaven Paré : Pour commencer, je voudrais que vous nous expliquiez comment vous en êtes arrivé à l'étude des robots, si ces recherches ont précédé votre intérêt pour le bouddhisme et comment ces deux domaines se sont rencontrés.

Masahiro Mori : J'ai commencé vers 1964. Voyez-vous, à l'origine, à cette époque, de nombreux jeunes exprimaient un sentiment de rejet du bouddhisme, mais pas moi, et ce, depuis tout petit. Au contraire, je me disais que j'aimerais étudier sérieusement le bouddhisme si l'occasion m'en était donnée. Et cette chance s'est présentée alors que j'avais commencé à créer des robots. Ce n'est qu'à ce momentlà que j'ai commencé à étudier sérieusement le bouddhisme. Voyez-vous, pour créer des robots, il faut avoir l'esprit ouvert. Et c'est pour cette raison, pour ouvrir les esprits, qu'a été créé le Mukta Research Institute ou «institut de recherche libre» - mukta est un terme sanscrit. Nous y pratiquions divers exercices destinés à nous ouvrir l'esprit.

C'est à cette période, lors d'une fête de fin d'année... enfin, normalement, lors d'une fête de fin d'année, on ne fait que boire et s'amuser, mais comme c'est très vite ennuyeux, nous avions décidé de nous rendre dans un temple et de pratiquer le zazen. 
- $\bullet$

3. Notion de liberté que l'on peut rapprocher de la liberté individuelle (NDT).
Le précepteur qui se trouvait là nous a interpellés : "Vous qui parlez d'“institut de recherche libre", savez-vous seulement ce qu'est la liberté? Vous devriez commencer par étudier cette notion fondamentale du bouddhisme! » C'est comme ça que tout a commencé.

Z.P.: En quelle année était-ce et quelles étaient les activités de cet institut de recherche libre, exactement? Pourriez-vous nous donner des détails sur sa création, par exemple?

M.M.: Vers 1970, lorsque j'ai commencé à étudier les robots, tout le monde autour de moi me disait : "Mais qu'est-ce que c'est que ces bêtises? Des jouets? » ou encore : "Ces recherches n'ont pas leur place dans une université! » Car tous avaient l'esprit fermé. À cette époque, les domaines des biotechnologies et des machines marchaient déjà main dans la main, mais lorsque j'évoquais de nouveaux rapprochements entre les biotechnologies et les machines, on me disait de changer de sujet de recherche. J'avais le sentiment que jamais je ne parviendrais à mes fins dans cet environnement, entouré par des gens à l'esprit si fermé. C'est pourquoi j'ai recruté des personnes qui avaient les mêmes vues et créé cet institut de recherche. Il comptait alors 28 personnes.

Chihiro Minato : Ce terme «liberté» (jizai en japonais) est celui de l'expression jiyuujizais, n'est-ce pas?

M.M. : C'est cela, c'est de là qu'il vient, le jizai de jiyuujizai. En anglais, on peut traduire jiyuu par freedom ou liberty, mais il n'y a pas de véritable équivalent pour le terme jizai. C'est pourquoi nous avons opté pour le terme sanscrit mukta.

Z.P. : Et après la création de cet institut?

M.M. : Eh bien, nous ne faisions pratiquement que des exercices destinés à nous ouvrir l'esprit.

\section{Z.P. : Concrètement?}

M.M. : Par exemple, nous faisions ce que nous pourrions appeler en termes modernes des brainstormings, et nous donnions également des conseils de guidance technique aux entreprises, ce qui nous permettait de payer le loyer de nos locaux. Et, bien entendu, lorsque nous entreprenions de nous y consacrer, nous étudiions le bouddhisme. Vraiment, nous faisions beaucoup de choses différentes. Nous avons par exemple créé ce serpent. Il est entièrement fait à la main. On a appelé cet animal mécanique Mechanimal (mechanic animal en abrégé). Il date de 1979 .

Z.P. : Vous l'avez fabriqué dans votre institut de recherche? Il fonctionne encore très bien.

M.M. : Oui. L'un des membres de notre institut souhaitait construire ce genre de chose, alors nous l'avons fait. Il peut se déplacer très rapidement.

Z.P. : De quel côté est la tête? (Rires.) 


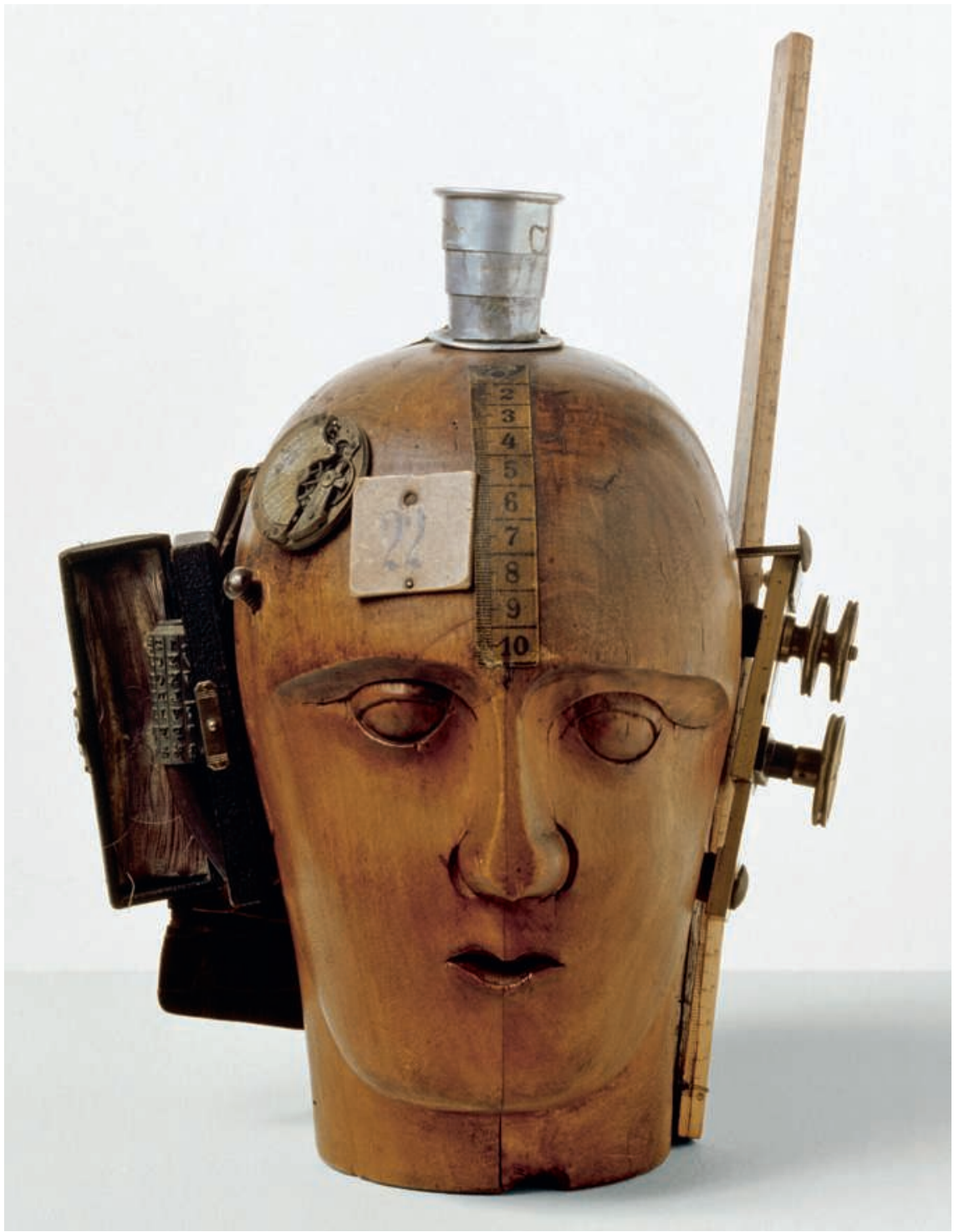

M.M. : Il fonctionne selon le principe du patinage : ses roues ne sont pas motrices. Il avance grâce à ce mouvement de va-et-vient, selon le même principe qu'un patineur. Et celui-ci est un myriapode.

Oh, les piles sont vides? Ou presque...

Z.P. : ... Ici, il est écrit « RoboCon" », il date de l'époque où le RoboCon a commencé?

M.M. : En fait, nous voulions le montrer lors du RoboCon, alors nous l'avons simplement décoré un peu.

Z.P. : Il a donc été créé avant. Combien avez-vous conçu de robots de ce genre?

M.M. : Oh, j'en ai construit tellement! Nous avions également fabriqué un crabe... Nous en avons créé un très grand nombre, vraiment!
Fig. 2 Raoul Hausman, L'Esprit de notre temps (Tête mécanique), 1919 () Centre Pompidou, MNAM-CCI, Dist. RMN/Philippe Migeat $\odot$ ADAGP, Paris 2012.
-

4. Abréviation pour « Robot Contest », un concours international de création de robots (NDT). 
-

5. Les caractères chinois ont généralement deux lectures en japonais (NDT).
Fig. 3 Bouddhas en bois doré du temple Sun lyu San Gen Do, Kyoto, mars 1962 @ Roger-Viollet.
C.M.: Pour en revenir au terme jizai, présent dans le nom de votre institut, pourriez-vous nous expliquer le sens que vous lui donnez?

M.M. : C'est un terme dont le sens est si profond, si complexe, que je doute qu'il soit possible de le traduire. Voyez-vous, en japonais, il peut être lu de deux façons ${ }^{5}$; l'une d'elles est mizukara aru, la conscience de soi. Mais une autre est onozukara aru, l'existence naturelle, factuelle. Le caractère " 自 " peut également se lire onozukara, donc jizai peut se lire onozukara aru. Ces deux notions qui à première vue peuvent sembler contradictoires composent l'essence de ce concept.

La conscience de soi serait-elle l'établissement de la personnalité ? C'est en tout cas son affirmation très nette.

C.M. : Lorsque vous dites onozukara, vous voulez dire "naturelle» ?

M.M. : Oui, comme dans "état d'esprit naturel, ouvert». Pour résumer, le véritable établissement de la personnalité, le corps... comment dire... il ne faut pas le confondre avec la notion européenne d'« ego "; en un mot, il faut fondre le soi et l'univers qui nous entoure. C'est pour cela qu'il faut étendre son être à tout l'univers, et c'est à cela que nous enjoint le bouddhisme. Et ceci est impossible tant que des barrières existent entre "soi » et «les autres». On ne peut pas parler de «liberté " au sens de jizai. Tant qu'un sentiment d'unité n'est pas présent, on ne peut pas parler de jizai.

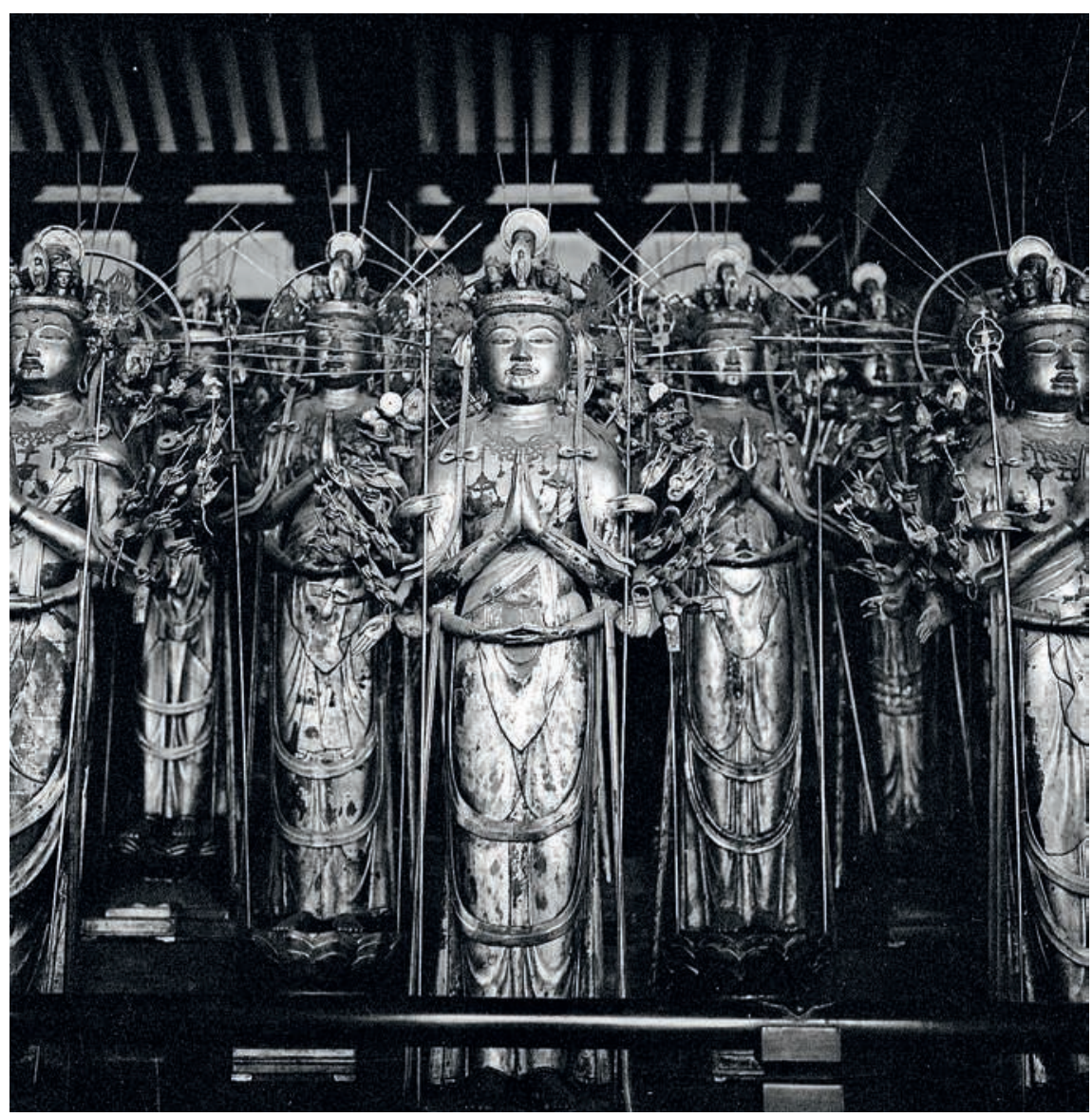


C.M. : Mais alors, dans les domaines scientifiques comme artistiques, si je ne me trompe pas, la séparation entre le créateur et la création, entre le soi et l'autre, entre le peintre et son modèle, etc., est toujours nettement marquée. Est-il impossible de parler de jizai à ce stade?

M.M. : Oui, impossible. C'est ce qu'enseigne le bouddhisme : cet état de division en deux entités n'est pas souhaitable. C'est pourquoi l'unité, la concentration de l'esprit sont nécessaires. Par exemple, tout le monde prend des photographies; lorsque l'on prend une photographie, on fait corps avec l'image que l'on voit dans le viseur : on oublie le "soi", on "devient" la scène que l'on photographie. On fait corps avec elle. C'est de cet état que je parle. Lorsque l'on fabrique quelque chose, on devient cette chose et uniquement elle. Je pense que vous avez compris. Lorsque vous créez quelque chose, vous devenez cette création. La sensation du soi disparaît. C'est cet état qui est le bon. C'est pourquoi le zazen est le moyen le plus efficace d'y parvenir.

Z.P. : J'aimerais en revenir au thème des robots. En est-il de même lorsque vous créez des robots?

M.M. : Tout à fait. Et pas seulement parce que créer un robot est une tâche qui requiert toute l'attention, mais également parce que le robot est une imitation de l'humain. Son modèle est l'être humain. Lorsque l'on veut concevoir un robot, avant de commencer, il faut bien comprendre l'être humain. Le bouddhisme, comme la robotique, sont deux approches de l'humanité.

Z.P. : Oui, elles sont semblables sur ce point. Je me suis demandé, en regardant ces deux robots que vous nous avez présentés, s'ils pouvaient nous aider à comprendre l'être humain et si les robots n'étaient pas une façon simple de montrer des choses complexes. J'ai aussi eu l'impression que des choses complexes sont simplifiées, et que cette simplification nous aide à les comprendre.

M.M. : Je suis tout à fait d'accord, en un sens. Mais d'un autre côté, lorsque l'on crée ces robots, on réalise également à quel point l'être humain est complexe.

Z.P. : En réalité, avec M. Grimaud, nous avons tous les deux lu ce livre (The Buddha in the robot), et il nous a fait une très forte impression. C'est la raison qui nous a conduits à venir vous déranger aujourd'hui! Dans ce livre, la théorie de l'uncanny valley n'est expliquée que très brièvement, sur à peine deux pages.

M.M. : Voudriez-vous en voir l'original? Le voici.

Z.P.: Oh! C'est vous, à l'époque de cet essai? Vous paraissez très dynamique! C'est la première fois que je vois le schéma original, qui est plus complexe que la version qui est généralement connue : sur la version la plus courante, ces deux courbes sont superposées et simplifiées.

M.M. : C'est ce que vous évoque ce schéma?

Z.P. : Oui, tout à fait. À côté de ce schéma, il y a des illustrations représentant des mains. Vous avez travaillé sur ce sujet dans les années 1970, n'est-ce pas? 
M.M. : J'ai commencé mes recherches sur les robots par les mains. Celle-ci compte trois doigts. Elle a été créée par une personne qui sculpte des mains de statues du Bouddha, c'est pourquoi elle est si réussie. Mais elle n'est pas mobile, il s'agit d'une simple maquette. Je vais vous montrer ma première création. Elle date de 1963. J'ai créé ces trois doigts et fait réaliser ce mécanisme pour son mouvement. Cette photographie a été prise à cette époque.

Z.P. : C'est un mouvement très complexe. Vos recherches pour la création de ce mécanisme et votre commande de sculpture de main...

M.M. : ... Ces deux événements se sont croisés fortuitement! Une simple coïncidence.

Z.P. : Je reviens un peu en arrière. À quelle occasion avez-vous élaboré la théorie de l'uncanny valley?

M.M. : Ça n'a pas été un moment en particulier... En fait, les statues de cire m'ont toujours mis mal à l'aise, tout simplement. On en voit souvent dans les expositions. Les regarder me met mal à l'aise, en particulier le visage et les mains. C'est la raison pour laquelle les statues de cire se trouvent à cet emplacement de la courbe. Ce sujet attire l'attention aujourd'hui, mais à l'époque, personne n'y avait prêté une grande attention. Ces derniers temps, tout le monde me pose des questions sur l'uncanny valley par-ci, l'uncanny valley par-là. (Rires.)

Z.P.: Un élément très intéressant de cette illustration, c'est ce corps, mort, en dessous de cette ligne. Cet élément inanimé, que rien ne peut mouvoir, complètement fixe et immobile, contraste avec ce qu'il y a au-dessus de la ligne, un élément qui va s'animer, un être vivant. Le sentiment de malaise provient-il de la relation entre l'élément immobile et l'élément animé?

M.M. : Eh bien, le mouvement générerait un malaise encore plus grand! Si ce corps mort bougeait, ce serait particulièrement dérangeant!

\section{Z.P. : Comme un zombie?}

M.M. : Exactement!

Fig. 4 Automate de Matsya, le premier avatar du dieu Vishnou prenant la forme d'un poisson. Son fabricant, Naikwadi, le décrit comme le plus hollywoodien des robots hindous jamais conçus. Photo Emmanuel Grimaud.
Z.P. : Aujourd'hui, la technique des nouveaux robots, enfin, les techniques les plus récentes, permettent de créer des mouvements très précis, d'exprimer des mouvements minutieux. Cela permet à des corps qui semblent «morts » d'avoir des expressions faciales, de mouvoir leurs yeux... Que pensez-vous de cela?

M.M. : Ça dépend des cas, il y en a de toutes sortes. À mon avis, certains robots fabriqués aujourd'hui se situent dans cette zone de l'uncanny valley, tandis que d'autres semblent en être déjà sortis.

Z.P. : Je voudrais vous poser une question à propos des statues du Bouddha que nous évoquions tout à l'heure... Ce schéma comporte aujourd'hui les statues du Bouddha. En tant que statues, ce sont des éléments parfaitement immobiles. De ce point de vue, on pourrait les rapprocher des corps morts... Cependant, pour- 


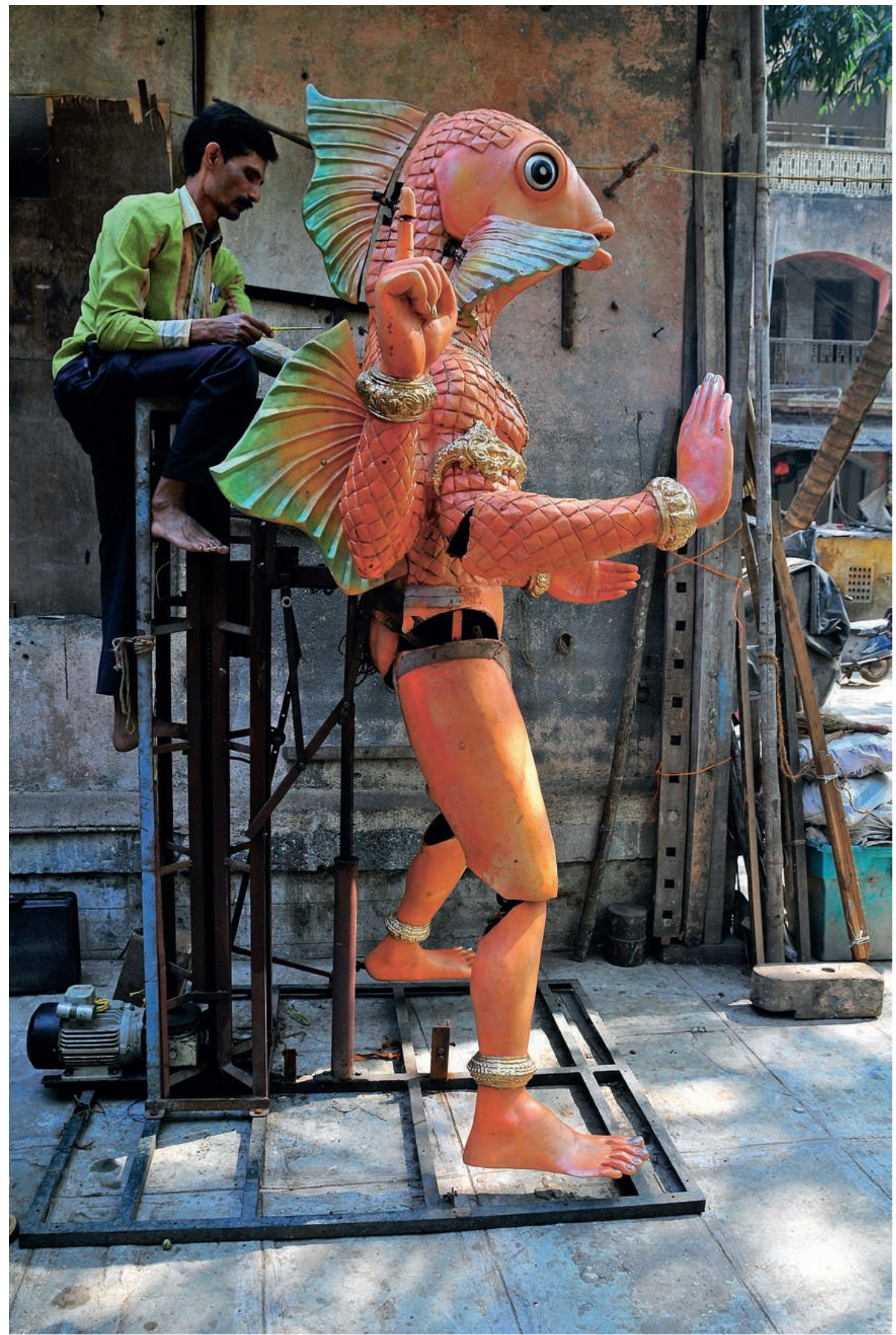




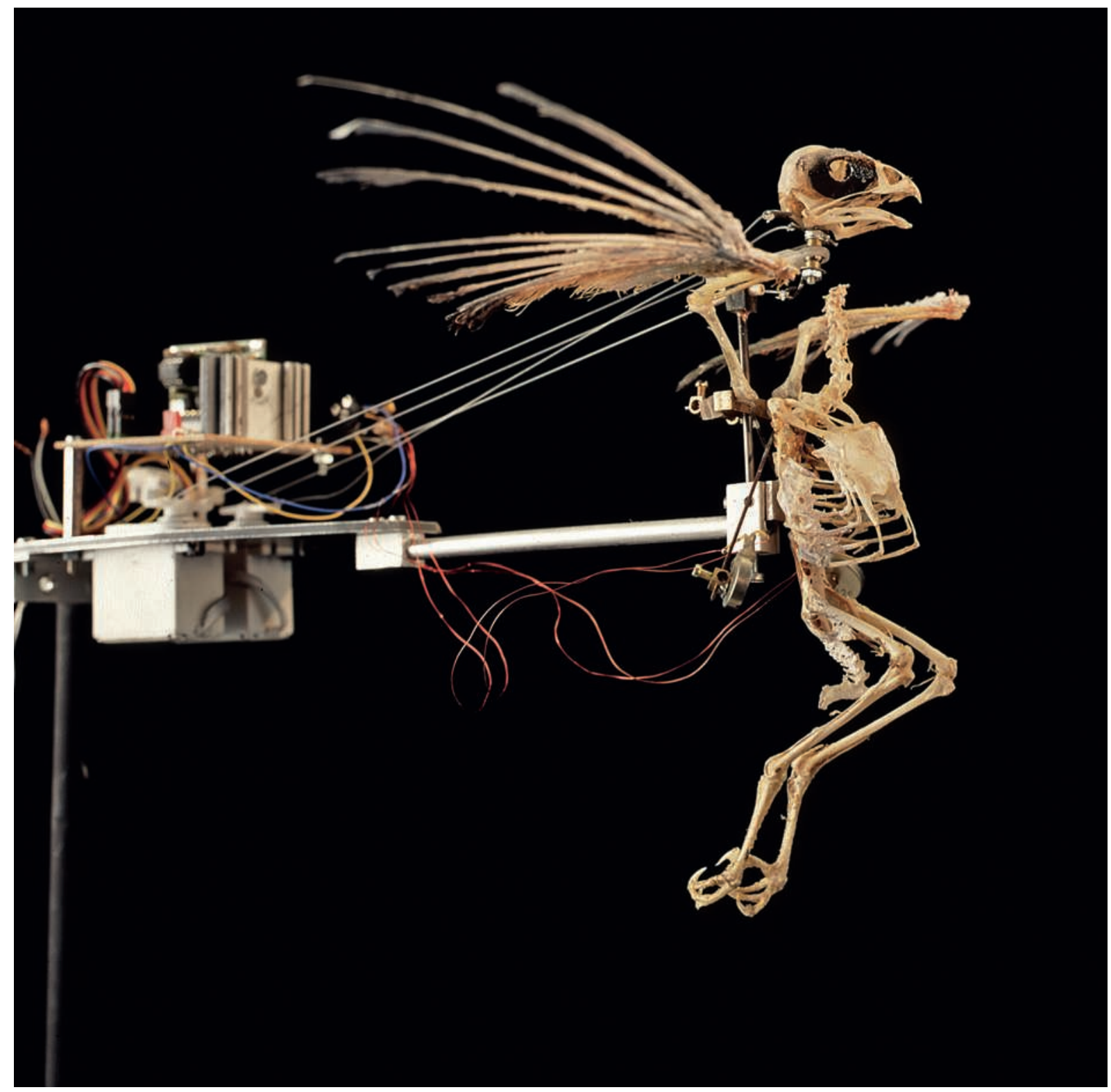

Fig. 5 Christiaan Zwanikken, Hovering Falcon, 2006. Avec l'aimable autorisation de Christiaan Zwanikken. quoi sont-elles placées en haut de votre courbe? Dans les ajouts que vous avez faits en 2005, les statues du Bouddha, comme le bodhisattva Maitreya, se situent sur la partie haute de la vallée.

M.M. : Et encore plus haut que la «personne en bonne santé », n'est-ce pas?

Z.P. : Pourquoi, alors qu'elles sont immobiles?

M.M. : Eh bien, parce que bien qu'elles ne puissent se mouvoir, ces sculptures inspirent la grâce de tout leur corps. Elles ont une aura de magnificence.

Z.P. : D'où vient cette magnificence? Nous voudrions en savoir plus. Par exemple, en tant qu'objets, elles sont incontestablement immobiles, mais ne pourrait- 
on pas comparer cette immobilité à l'instant qui précède le mouvement? Nous avons également visité le temple Sanjūsangen-dō ${ }^{6}$, à Kyoto, et nous avons été frappés par le fait que chaque statue possède un visage différent.

M.M. : Oui, chaque visage est différent.

Z.P. : En les regardant, nous avions l'impression qu'elles allaient se mettre à bouger d'un instant à l'autre, ou alors que leur fixité emprisonnait un mouvement.

M.M. : Ces sculptures donnent vraiment l'impression de contenir le mouvement à l'intérieur de leur immobilité. Elles dégagent également cette impression, non?

Z.P. : D'une certaine façon, oui. Parfois ça opère et parfois non, mais comme vous le dites, lorsque ça fonctionne, une aura s'en dégage.

M.M. : Vous aimez Maitreya?

Z.P. : En fait, nous nous sommes référés aux trois sculptures du Bouddha que vous citez dans votre ouvrage. Est-ce parce qu'elles vous plaisent particulièrement, ou aviez-vous une raison plus précise de les prendre comme exemples?

M.M. : En fait, il y a de nombreuses statues du Bouddha qui me plaisent. J'ai mis en avant celles du Sanju-Sangen-Do, Maitreya et...

Z.P. : ... Aussi d'autres temples de Nara et de Kyoto, l'une du temple Kofuku-ji...

M.M. : ... Le temple Kofuku-ji! J'ai toujours apprécié la courbe très marquée des sourcils de la tête de son Bouddha. L'une des particularités des statues du Bouddha, c'est l'absence de pli, ici, entre les sourcils. Cet endroit semble toujours receler quelque chose de scintillant. Et après une séance de méditation zen, notre visage présente cette même expression. En tout cas, moi, j'ai cette impression quand cette marque disparaît.

Z.P. : Maitreya semble être parmi les humains et les contempler, tandis que Gakko, regardant la lune...

M.M. : ... Gakko! Il s'appelle bodhisattva Gakko?.

Z.P. : C'est ça, Gakko semble avoir le regard tourné vers le ciel.

On pourrait dire que l'un des thèmes les plus importants pour les roboticiens ou les chercheurs en robotique, en particulier ceux qui travaillent sur des modèles humanoïdes, est celui du regard.

M.M. : Oui, c'est un thème très important.

Z.P. : Pourriez-vous nous dire ce que vous en pensez, en particulier parce que les statues du Bouddha ont les yeux fermés...

M.M. : ... Entrouverts, pas fermés, en réalité. Savez-vous pourquoi on entrouvre les yeux? S'ils étaient ouverts, on verrait des choses qui créeraient des pensées

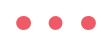

6. Pavillon des Trente Intervalles, appelé aussi temple des Mille Bouddhas (NDT).

7. Candraprabha, «clarté de la lune » (NDT). 
parasites. Et s'ils étaient fermés, on finirait par s'endormir. C'est pourquoi le zazen se pratique les yeux entrouverts.

C'est également le meilleur moyen de se relaxer.

Lorsque je regarde les statues du Bouddha, je les vois les yeux entrouverts, en particulier lorsqu'elles sont assises pour pratiquer le zazen.

Z.P. : Avez-vous travaillé particulièrement sur le mouvement des yeux des robots que vous avez créés?

M.M. : Non, pas encore, j'en suis encore loin. Mes dernières recherches ont été focalisées sur le déplacement bipède.

Que ce soit du point de vue de la reconnaissance optique (pattern recognition) ou selon celui que nous avons évoqué - la noblesse des traits des statues du Bouddha-, je ne pense pas que des recherches sur ce mouvement particulier des yeux soient menées actuellement. En tout cas, les roboticiens japonais n'ont pas encore tourné leur regard dans cette direction. (Rires.)

Z.P. : La recherche peut-elle s'orienter dans cette direction, à votre avis?

M.M. : Je pense que ce serait bien, il y a forcément des gens que cela intéresse.

Z.P. : On dit que la robotique japonaise est la plus avancée en termes de robots humanoïdes. En prenant en compte votre travail jusqu'à aujourd'hui, dans quelle direction pensez-vous que devrait s'orienter la recherche?

M.M. : Le nombre de roboticiens a énormément augmenté récemment. Dernièrement, lors d'une conférence scientifique consacrée à la robotique, trois jours ont été nécessaires à la présentation de près de 800 dossiers. L'association des roboticiens compte près de 4000 membres, ce qui est considérable. Et, en réalité, seule une infime partie des robots trouve un usage pratique, la plupart en tant que robots industriels. Alors que nous avons besoin de robots capables de fournir une aide à la personne, aucun n'a encore pu être réalisé. C'est sur ce secteur qu'il y a urgence, à mon avis.

Z.P. : Dans ce domaine, le contact humain devient extrêmement important.

M.M. : Tout à fait.

Puis-je vous offrir une tasse de thé?

Z.P. : Merci, avec plaisir.

M.M. : Pardonnez-moi, ma femme est sortie.

C.M. : Mon oncle était un peintre japonais qui habitait près d'Osaka. Il peignait des tableaux représentant des scènes de bunraku. Quand j'étais petit, il m'emmenait voir des représentations de bunraku, et je voyais très souvent ses tableaux. Mais les marionnettes de bunraku m'effrayaient.

Assistez-vous à des représentations de bunraku?

M.M. : Oui, je connais. Mais honnêtement, je ne trouve pas les marionnettes de bunraku particulièrement dérangeantes. 
Z.P. : Moi non plus, aujourd'hui. Et je trouve qu'elles ont parfois des traits plus humains que les humains.

M.M. : En réalité, pour en revenir à l'uncanny valley, c'est un sujet qui recouvre des thèmes très denses. Ce qui est intéressant, c'est qu'en l'étudiant, les gens peuvent y trouver des significations très profondes. En vérité, la problématique dépasse la simple question: "La ressemblance est-elle souhaitable?" Par exemple, les marionnettes de bunraku ne ressemblent pas vraiment à des êtres humains. Et pourtant, cela fonctionne bien. Pourquoi? Parce qu'elles bougent? Parce qu'elles sont capables d'expressions faciales? C'est une question à laquelle il n'y a pas de réponse, je pense.

Z.P. : Effectivement. Qu'est-ce que la ressemblance? J'aurais aussi des difficultés à la définir.

M.M. : Oui, c'est plus une question philosophique que de robotique. C'est la même question que "que signifie être identique?».

Connaissez-vous le conte folklorique japonais Hana-Saka-Jijii ${ }^{8}$ ? Dans cette fable, le chien aboie pour indiquer les endroits où il faut creuser. Eh bien, je suis comme ce chien. Je pense que je possède ce flair qui me fait renifler le trésor. En revanche, je ne semble pas doué pour creuser moi-même. J'aboie, j'aboie en permanence, pour dire «Creusez par ici!». Mais au départ, j'avais beau aboyer, personne n'y faisait attention. Du coup, j'ai bien été obligé de creuser un petit peu moi-même.

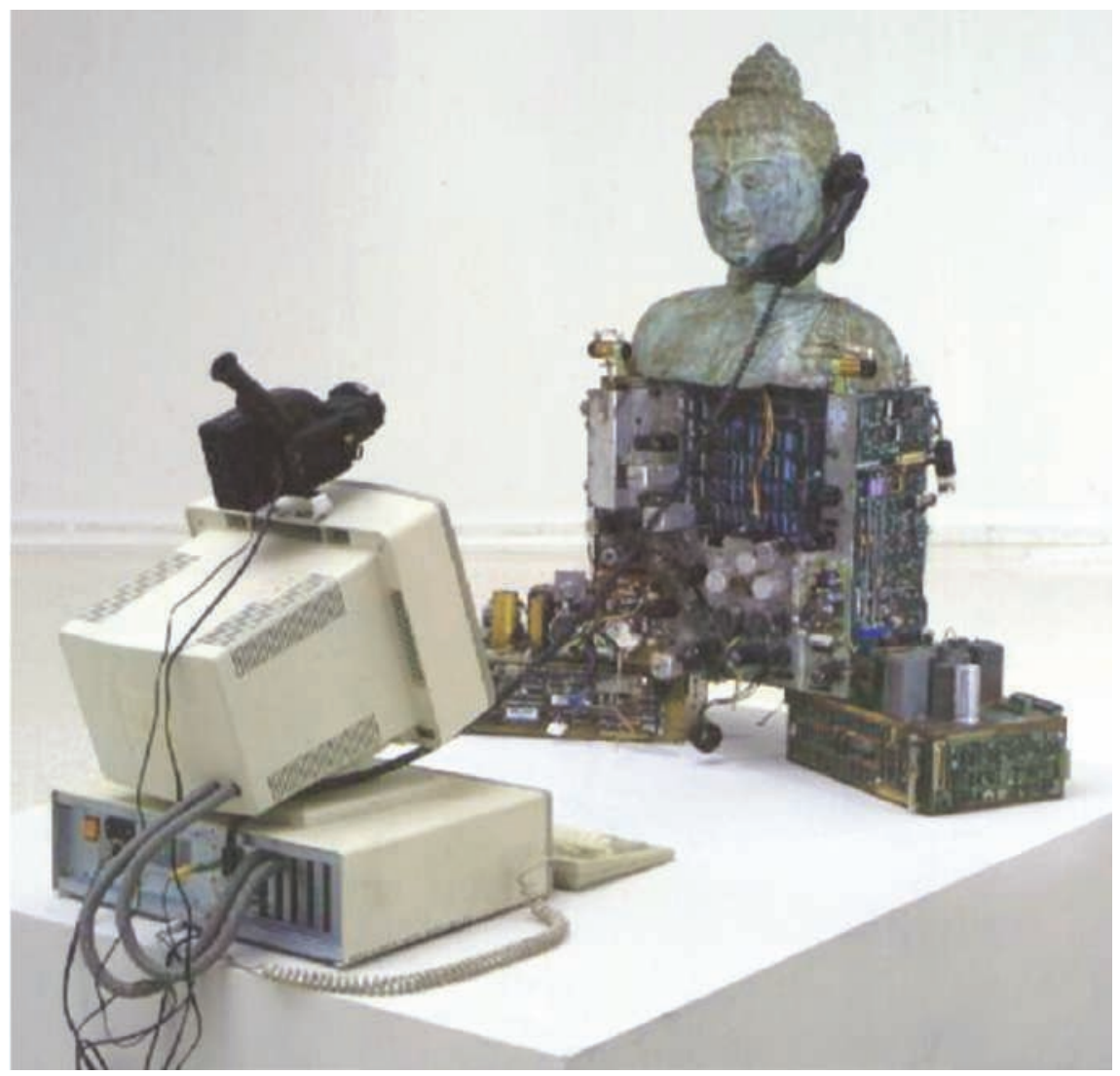

(

8. Littéralement «le vieil homme qui fait pousser les fleurs », parfois traduit par «le vieillard qui faisait revivre les arbres morts 》 (NDT).
Fig. 6 Nam June Paik, Techno-Buddha, 1994 (c) Nam June Paik, DR. 
Et j'ai découvert un petit morceau du trésor. C'est alors que tout le monde s'est précipité pour creuser aussi, comme une véritable ruée vers l'or. C'est cela, l'histoire de l'uncanny valley. Oui, c'est vraiment ça. Ce que j'ai fait jusqu'à maintenant, c'est poser habilement la problématique, mais je ne suis pas doué pour poursuivre la recherche de la solution. Je pense que cela définit bien mon caractère.

Z.P. : Eh bien, par exemple, lorsqu'on lit des essais ou des articles ayant trait à l'uncanny valley publiés tout autour du monde, on a l'impression que la plupart tiennent pour "dérangeant » l'ensemble du corps du robot, ou bien parfois l'ensemble du visage, sans aller plus loin. Mais si, en réalité, l'important était le mouvement des différents éléments, les mouvements précis de chaque partie?

M.M. : Oui, je suis tout à fait d'accord avec vous. Les sculpteurs de statues du Bouddha disent que les dernières finitions sont les plus dangereuses, le dernier coup de ciseau est le plus risqué. Une erreur, et c'est le Maitreya qui est raté. C'est vraiment très difficile. Il s'agit d'effectuer la dernière touche autour des yeux sans rien enlever de trop, sinon c'est fichu.

Z.P. : Cela me rappelle cette histoire d'une femme de la cour impériale qui fit fabriquer un masque à l'image de son amant. Elle souhaitait conserver son image pour toujours. Mais lorsque le masque fut terminé, elle le regarda et finit par le rejeter. Le degré de finition, de ressemblance, avait donné au visage sculpté un aspect vivant qu'elle n'avait pas prévu. Ce qui provoque le malaise, peut-être, n'est pas l'impression globale produite, mais plutôt... comment dire... la finition de chaque partie, l'harmonie créée par l'ensemble des parties, par les détails?

M.M. : J'ai travaillé à l'université de technologie de Tokyo, et l'une de nos recherches portait sur la beauté. J'ai donc demandé à l'Université des femmes de prendre des photos de toutes les élèves, et je les ai comparées en détail, cherchant à déterminer quels étaient les plus beaux yeux, la plus belle bouche, en effectuant une sélection pour assembler le plus beau visage. Mais le résultat fut loin d'être «beau ». C'est une histoire étrange, n'est-ce pas?

Cela avait surpris tout le monde. Il semblerait que la symétrie parfaite ne fonctionne pas. Un certain degré d'imperfection, d'asymétrie, semble nécessaire. C'est un point très difficile à atteindre.

Z.P. : Mais alors, d'où provient la beauté parfaite, le visage de femme parfait?

M.M. : C'est difficile à dire, je ne saurais pas le définir. Nous avons également fait une autre expérience. Lorsque l'on sourit, on effectue ce mouvement avec les muscles du visage. Nous avons filmé ce mouvement et l'avons regardé au ralenti, très lentement. Eh bien, les étapes intermédiaires menant au sourire sont des visages extrêmement dérangeants. Bien que le sourire soit charmant, les expressions intermédiaires du processus sont dérangeantes, comme des visages que l'on ne devrait pas regarder.

En fait, l'important, c'est la vitesse. Il faut que le mouvement se déroule à la bonne vitesse.

Z.P. : À propos de sourire, de visage souriant... Les statues du Bouddha, pour la plupart, sont souriantes. Que vous inspire ce sourire? 


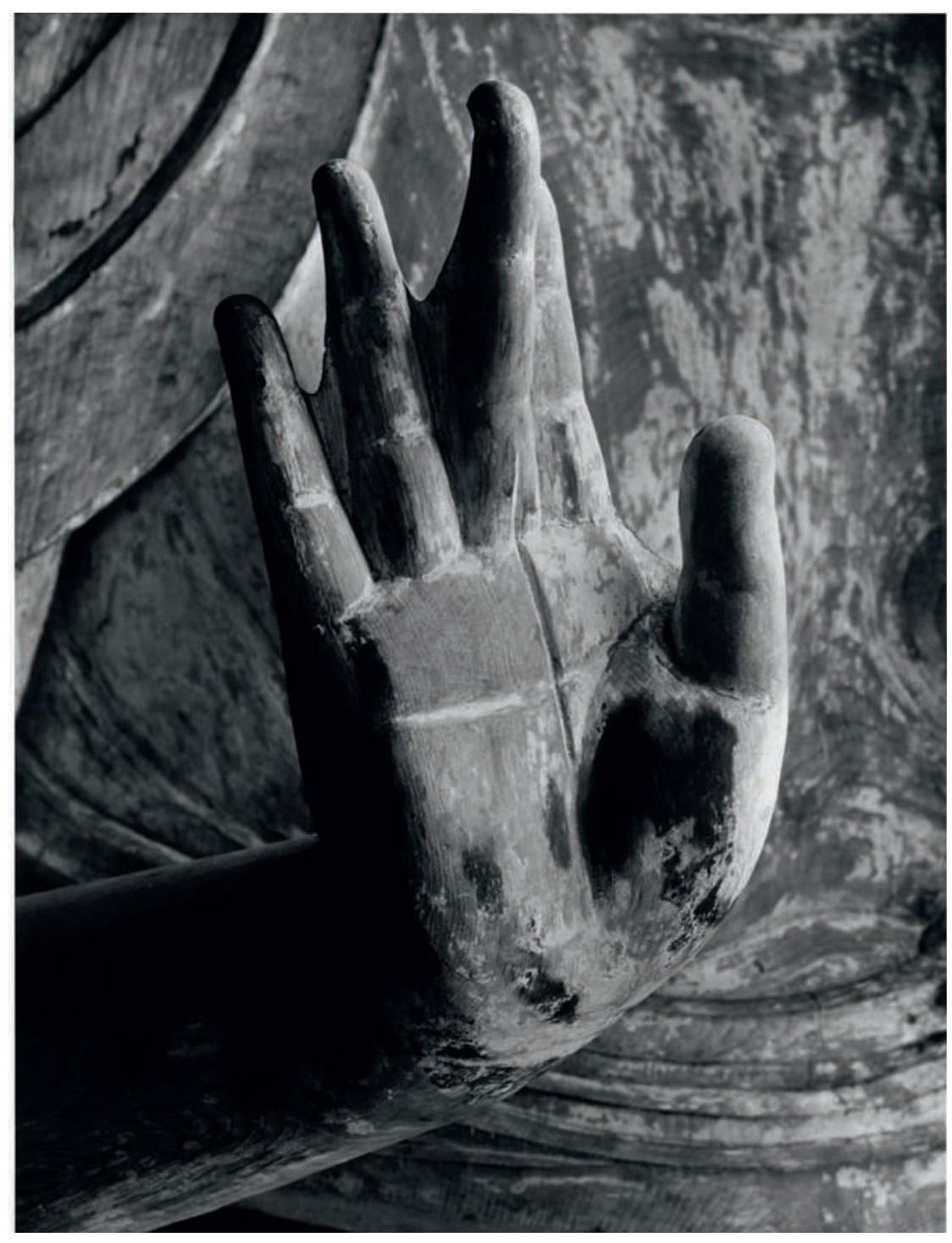

M.M. : Prenons un exemple : dans le temple Chuuson-ji... ou plutôt une statue du temple Horyu-ji qui a un très beau sourire, avec les joues comme ceci. C'est très beau... Comment dire... c'est une statue debout, en pied, il me semble... Où se trouve-t-elle déjà?

Les statues du Bouddha semblent détachées du monde. Elles semblent heureuses d'être détachées du monde.

... C'est celle du temple Chuuguu à Horyu-ji, c'est bien à ce Maitreya que je pense.

Z.P.: Oui, c'est Maitreya. Il se trouve dans ce temple au sud de Nara... La position de sa main, en particulier, est très intéressante, comme si son mouvement était suspendu. Cette suspension, plus que le mouvement lui-même, semble attirer l'attention. C'est une position très délicate et subtile. Comme si elle hésitait à toucher... Plus que le simple mouvement, c'est cette subtilité qui renferme quelque chose de fascinant...

M.M. : ... Ici, n'est-ce pas? Autour de la bouche, c'est très beau.

Z.P.: On pourrait dire que cette expression est l'une des plus belles réussites de l'art bouddhique japonais, même si cette statue date quelque peu, non?
Fig. 7 Ken Domon, Main droite de l'image assise du bouddha Shakyamuni dans le hall de Miroku, Muro-Ji, Nara (Japon), 1942-1943. Don de l'artiste, $n^{\circ}$ inv. 319.1976 @ Ken Domon Museum of Photography. 


\section{ENTRETIEN}

ROBOTS ÉTRANGEMENT HUMAINS

Fig. 8 Kenji Yanobe,

Viva Riva Project - Standa, 2001.

Photographie prise au BTAP

Beijing Tokyo Art Projects Gallery,

Pékin, 2007. Avec l'aimable

autorisation de la Yamamoto

Gendai Gallery/photo Edward

Sanderson.

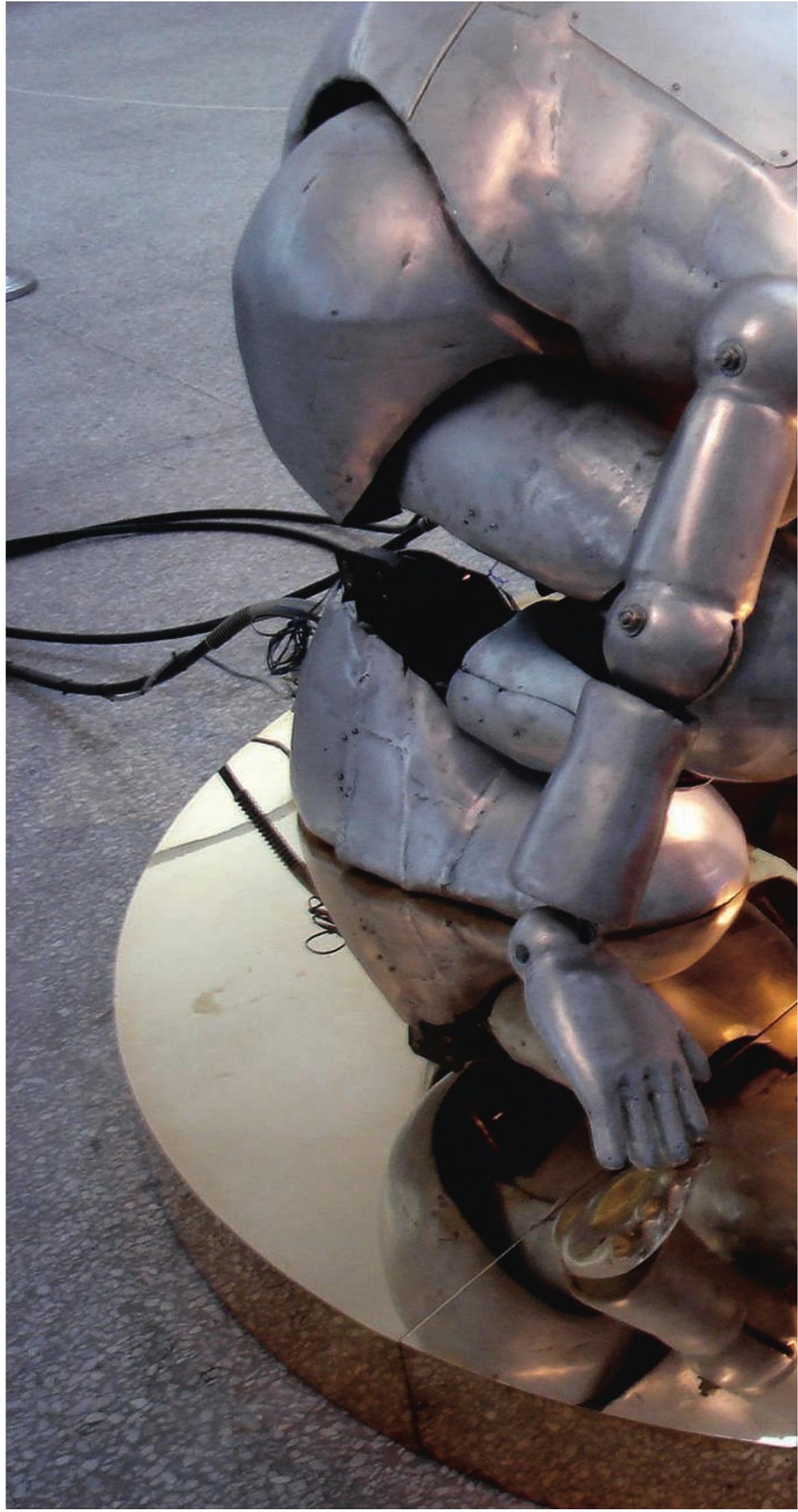


M.M. : Et peut-être même encore plus pour la raison qu'elle est ancienne. On ne pourrait plus en faire de semblable, aujourd'hui.

Z.P. : Dans l'art occidental également, à une certaine époque de la Grèce antique, on trouve des statues d'hommes ou de dieux qui portent une expression qu'on ne saura plus reproduire ensuite. C'est un phénomène similaire.

En regardant les recherches en robotique actuelles, on peut constater que l'on ne parvient pas à saisir de tels détails du visage humain.

M.M. : Bien entendu. Je me demande si ce ne sont pas les progrès de la civilisation qui nous font perdre ce savoir-faire...

\section{Z.P. : ... D’une façon générale?}

M.M. : Oui, d'une façon générale. Pour sculpter une statue du Bouddha, on commence par méditer en zazen. Ensuite, avant chaque coup de ciseau, on effectue un salut et une prière, puis on peut procéder à un autre coup de ciseau... Sans se rapprocher soi-même du Bouddha, on ne peut pas sculpter de statue du Bouddha.

Z.P. : Les deux animaux mécaniques que vous nous avez montrés tout à l'heure, en particulier le serpent, ont un côté très poétique, dû certainement à leur simplicité. Et nous avons l'impression que la complexification des robots leur fait perdre cet aspect poétique. Avez-vous retrouvé ce côté poétique parmi les robots plus récents que vous avez pu voir?

M.M. : Hum, pas particulièrement, non... Mais je dois dire qu'Asimo est bien plus facile à regarder que la plupart des robots humanoïdes dotés de visages humains. On peut le regarder sans ressentir de répulsion.

Z.P. : Si Asimo est plus facile à regarder, pourriez-vous définir d'où vient la répulsion engendrée à la vue de robots humanoïdes fabriqués pour ressembler le plus possible à un être humain?

M.M. : C'est ma théorie, mais je serais incapable de l'expliquer! (Rires.)

Z.P. : Connaissez-vous le robot $\mathrm{CB}_{2}{ }^{9}$ ? Celui qui ressemble à un bébé.

M.M. : Oui, mais je ne l'aime pas tellement.

Z.P. : J'ai été très surpris de voir un robot [Asimo] qui semblait tellement poli et qui respectait les codes de conduite sociale. Pensez-vous qu'il est nécessaire que les robots soient plus polis que les humains? Par exemple, les robots destinés au service à la personne devraient-ils être plus polis qu'un être humain?

9. Robot humanoïde du laboratoire Erato de l'université d'Osaka.
M.M. : Mais c'est simplement une question de programmation! Contrairement à un être humain auquel on enseigne des règles, le robot ne peut pas aller à l'encontre de son programme. C'est facile! (Rires.)

Z.P. : Sur le sujet de l'uncanny, dans le cas d'Asimo... Il ne dispose pas d'expression faciale, il peut juste mouvoir ses membres, etc., mais il est doté de la parole. Que pensez-vous du rôle de la parole, de la voix, chez le robot? 


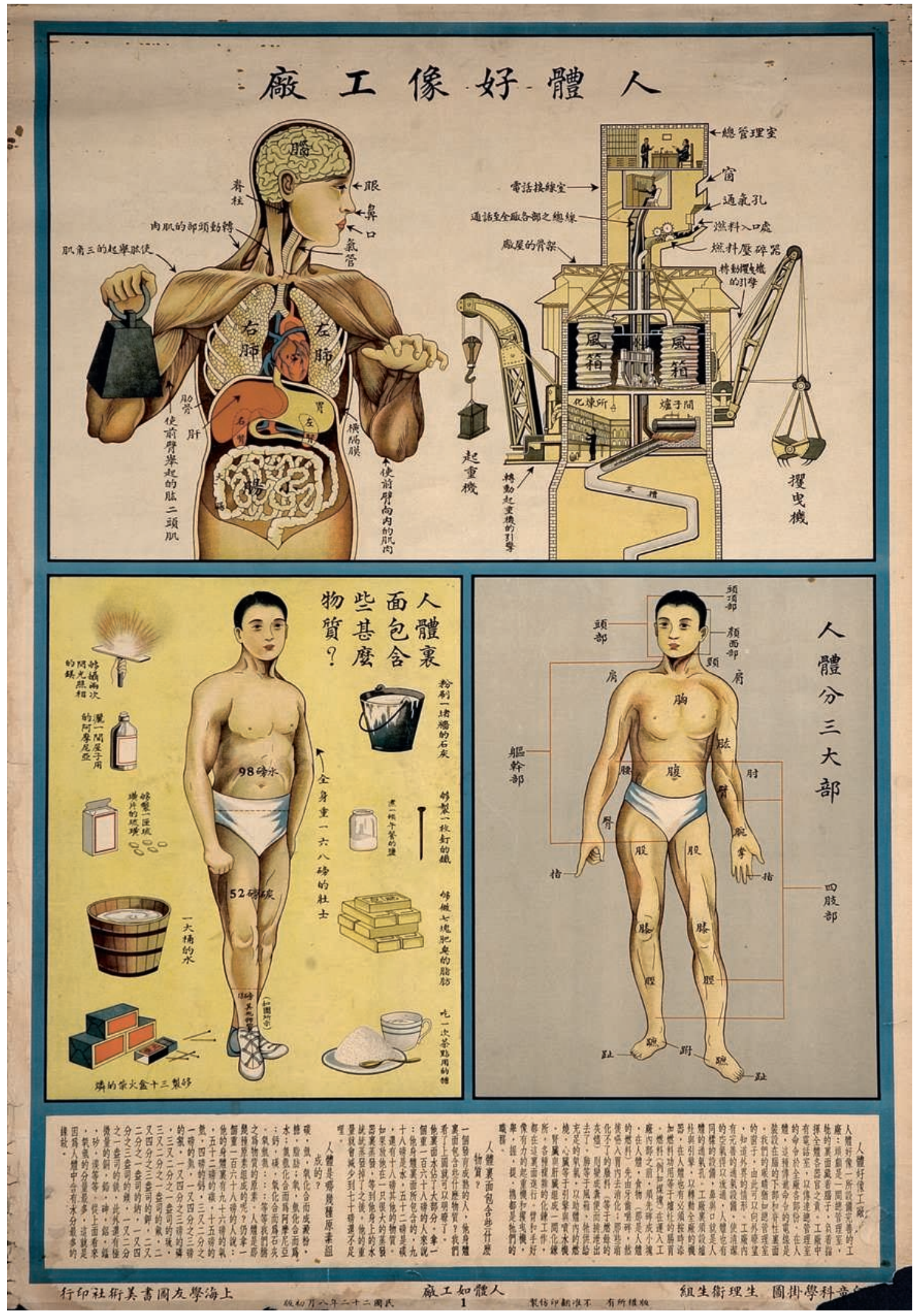

Fig. 9 Dessin anatomique chinois : le corps humain est comme une usine, 1933. Avec l'aimable autorisation de la National Library of Medicine, Bethesda, Maryland. 

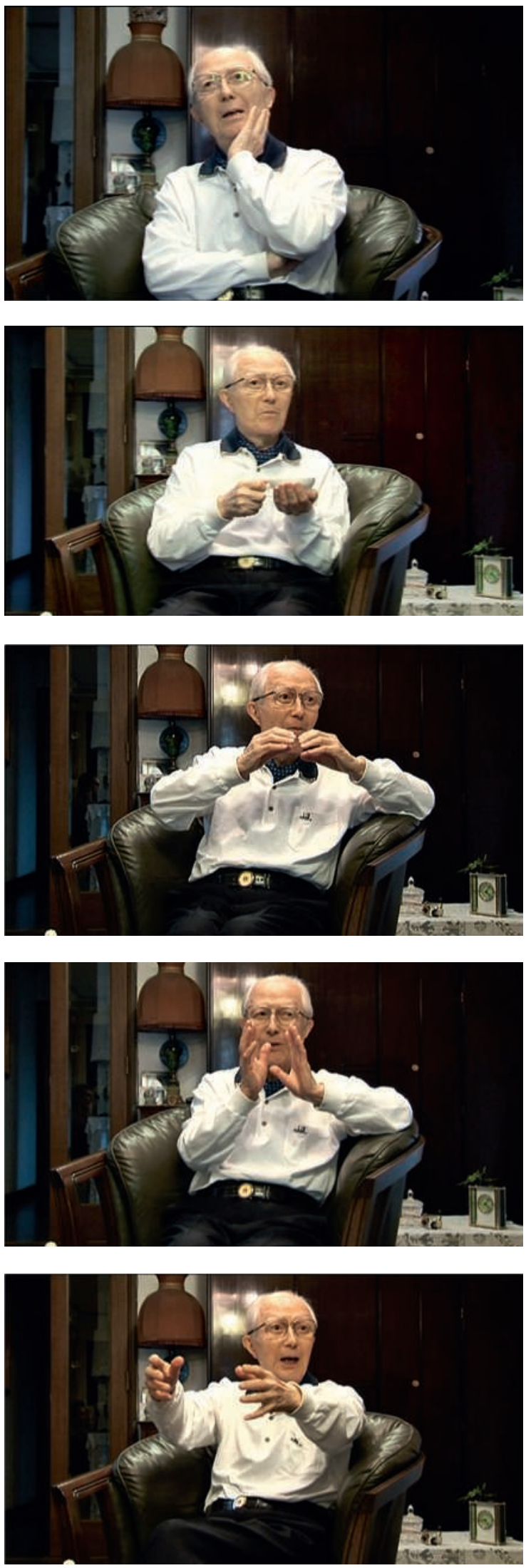

M.M. : En fait, il est doté d'une "voix de robot". La voix d'Asimo est composée d'enregistrements de voix humaines, alors c'est un peu plus facile, mais... Était-ce dans les années 1970? En quelle année s'est déroulée l'Exposition internationale de Tsukuba? Ah oui, 1985.

Donc, en 1985, j'ai également présenté un robot. J'ai demandé si quelqu'un dans le public voulait essayer de parler avec le robot, et j'ai invité un jeune garçon à le faire. Il avait levé la main pour montrer qu'il voulait essayer et il s'était avancé. L'hôtesse qui faisait la présentation lui a demandé d'où il venait. Je vais devoir entrer un peu dans les détails: dans les environs de Tokyo, à Chiba, il y a un endroit appelé Ichikawa. Eh bien, le garçon a répondu "I-CHI-KA-WA»! Tout le monde a éclaté de rire : il était tellement dans l'idée de parler au robot que lui aussi avait modifié sa prononciation pour prendre une "voix de robot " pour dire « I-CHIKA-WA " plutôt qu' «Ichikawa ». Il avait pris une voix de robot. Comme si cette intonation caractéristique était la marque d'une voix de robot.

\section{Z.P. : Quel âge avait-il environ?}

M.M. : Un élève d'école primaire.

Z.P. : Dans les années 1960, vous avez travaillé sur des robots industriels. À cette époque, les appareils électroniques ménagers étaient déjà dotés de voix, mais pratiquement aucun robot de chaîne de montage.

M.M. : Non, ceux-là ne l'étaient pas. Parce que la communication vocale est plus onéreuse.

Z.P.: Aujourd'hui, surtout au Japon, la plupart des machines parlent, comme les GPS par exemple. Mais ceci reste cantonné au niveau domestique, civil, tandis que les robots industriels restent muets.

M.M. : Eh bien, tant que c'est le robot qui parle et l'humain qui écoute, cela reste possible. Mais si un humain parlait à un robot pour lui donner des instructions, cela pourrait être très dangereux. Cela poserait un problème de précision et d'exactitude.

C.M.: On trouve deux sortes de temples au Japon, les temples bouddhistes et les temples shinto, n'est-ce pas? Pour des raisons historiques, c'est très compliqué, mais par exemple, dans les temples shinto...

Fig. 10 Interview de Masahiro Mori, photogrammes Emmanuel Grimaud. 
M.M. : ... Le terme pour « temple shinto » est jinja.

Z.P.: Oui, les jinja. Dans les temples shinto, on ne voit presque pas de représentation humaine. Que pensez-vous de cette différence entre le shintoïsme et le bouddhisme?

M.M. : Je pense que le shintoïsme est centré sur le culte des ancêtres, tandis que le bouddhisme se concentre plus sur la recherche de la vérité et la dévotion. Mais je ne suis pas très instruit en ce qui concerne le shintoïsme.

Z.P. : Mais on dirait que le shintoïsme est détaché de l'homme, en tout cas de la représentation humaine.

M.M. : Ou plutôt les choses doivent rester hors de la vue.

Z.P. : Oui, c'est ça. Nous sommes allés au grand temple d'Ise au moment d'un senguu ${ }^{10}$, mais nous n'avons rien pu voir.

M.M. : Oui, cela doit rester caché. D’autre part, dans le shintoïsme, on peut recevoir un châtiment divin, tandis que cet aspect n'existe pas dans le bouddhisme. Oui, l'idée de compassion est contraire au principe de la punition.

C.M. : Depuis tout petit, je me demande d'où cela provient, cette idée de châtiment.

M.M. : Dans le bouddhisme, les statues du Bouddha ne sont que des instruments transitoires. Bouddha est la vérité de l'univers, il est l'incarnation humaine de la vérité de l'univers. Il n'a rien d'idolâtre, tout comme le christianisme qui a combattu l'idolâtrie.

Z.P. : En ce sens, les robots pourraient-ils être des formes transitoires, comme les statues du Bouddha? (Rires.)

M.M. : Une forme transitoire de l'homme? Rien n'empêche de l'imaginer ! Je vais vous raconter une histoire. Je reviens un peu en arrière. Elle se passe dans un temple très pauvre, par une froide journée d'hiver. Un voyageur fait irruption dans ce temple et demande à y passer la nuit pour s'abriter du froid. Le précepteur du temple lui répond que le temple est très pauvre, qu'il ne dispose même pas de bois pour faire du feu. Mais, devant la détresse de l'homme, le précepteur débite une statue du Bouddha et la jette dans le feu pour les réchauffer. Cela surprend grandement le voyageur, mais le précepteur n'en fait aucun cas. C'est plutôt cela, l'esprit du bouddhisme.

Z.P. : Alors on pourrait aussi, en cas de besoin, démonter un robot.

M.M. : Oui! (Rires.)

Z.P. : Eh bien, merci beaucoup.

M.M. : Je vous en prie, mais je ne pense pas vous avoir été d'une grande aide.
-

10. Transfert d'un objet abritant une déité (NDT). 